\title{
Ansiedade materna e maternidade: Revisão crítica da literatura
}

\author{
Mariana Reichelt Chemello \\ Daniela Centenaro Levandowski \\ Tagma Marina Schneider Donelli
}

\section{RESUMO}

Este artigo apresenta uma revisão sistemática da literatura sobre ansiedade materna e maternidade, publicada entre janeiro/2009 e dezembro/2014. Foram examinados 43 artigos cujo foco de investigação era a ansiedade da mãe, a partir de consulta às bases de dados American Search Premier Complete, LILACS, MEDLINE, PsycINFO, SciELO e Web of Science. O material foi analisado a partir das categorias ano da publicação, idioma, área da publicação, delineamento, participantes, instrumentos, objetivos e principais resultados e conclusões. Verificouse a necessidade de desenvolvimento de instrumentos de medida específicos para a ansiedade materna, adaptados à realidade brasileira, e a importância da sua identificação no período pré-natal, como medida preventiva e protetiva para a saúde mental das mães, o desenvolvimento dos bebês e a qualidade da relação da díade. Os resultados também sugerem a necessidade de novos estudos que possam avaliar diferentes intervenções e tratamentos para o manejo dessa condição.

Palavras-chaves: Ansiedade, ansiedade materna, maternidade, relação mãe-bebê, revisão sistemática.

\section{ABSTRACT}

\section{Maternal anxiety and motherhood: Systematic review of the literature}

This article presents a systematic literature review about maternal anxiety and motherhood, considering the period between January 2009 to December 2014. Forthy-three scientific articles, focused on mother's anxiety, were included, derived from a search to the following databases: American Search Premier Complete, LILACS, MEDLINE, PsycINFO, SciELO and Web of Science. The analysis was based on year of publication, language, area of publishing, design, participants, instruments, objectives and main results and conclusions. The need to develop instruments specifically targeted to assess maternal anxiety in Brazilian context was noted, and also the importance of its identification during the pregnancy, as a preventive and protective measure for the mothers mental health, the babies development and the quality of this dyadic relationship. The findings pointed to the need for further studies to evaluate different interventions and treatments to the management of this psychological condition.

Keywords: anxiety; maternal anxiety; maternity; mother-baby relationship; sistematic review.

A ansiedade é uma vivência comum, potencialmente típica e experimentada por qualquer ser humano e se caracteriza por um sentimento difuso, desagradável e vago de apreensão, como inquietação, desconforto e taquicardia. A ansiedade é uma resposta a uma ameaça desconhecida, interna, vaga ou de origem conflituosa (Kaplan, Sadock, \& Grebb, 1997). Assim, essa vivência faz parte das experiências humanas e é um estado emocional esperado e necessário para a sobrevivência, pois, na sua ausência, o ser humano não lutaria, não procuraria vencer obstáculos e nem fugiria de situações perigosas (Barbirato \& Dias, 2009; Gorenstein, Andrade, \& Zuardi, 2000; Kaplan \& Sadock, 2000; Rodrigues, 2011).

Todas as pessoas, em algum momento da vida, experimentam a ansiedade. No entanto,

\section{Sobre os Autores}

M.R.C.

orcid.org/0000-0002-9210-1751

Universidade do Vale do Rio dos

Sinos (UNISINOS) - São

Leopoldo, RS

marianareichelt@gmail.com

D.C.L.

orcid.org/0000-0002-6338-7287

Universidade Federal de

Ciências da Saúde de Porto

Alegre (UFCSPA) - Porto Alegre, RS

danielal@ufcspa.edu.br

T.M.S.D.

orcid.org/0000-0003-3083-0083 Universidade do Vale do Rio dos Sinos (UNISINOS) - São Leopoldo, RS

tagmad@unisinos.br

\section{Direitos Autorais}

Este é um artigo de acesso aberto e pode ser reproduzido livremente, distribuído, transmitido ou modificado, por qualquer pessoa desde que usado sem fins comerciais. 0 trabalho é disponibilizado sob a licença Creative Commons CCBY-NC. 
quando ela é excedente, em vez de contribuir para a adaptação, acaba por desencadear a falência da capacidade adaptativa do indivíduo (Benute, Nomura, Pereira, Lucia, \& Zugaib, 2009). Particularmente, no contexto da maternidade, a ansiedade pode ocorrer em diferentes situações e contextos. Um estudo evidenciou que as mães experimentam sentimentos ambivalentes em torno dessa experiência, uma vez que, por um lado, sentem-se muito felizes e apaixonadas por seus bebês, embora também seja comum que se sintam extenuadas e ansiosas (Rapoport \& Piccinini, 2011). Outro estudo demonstrou que quadros de ansiedade são muito frequentes durante o ciclo gravídico-puerperal; estima-se que $20 \%$ das mulheres apresentam sintomas de ansiedade durante a gravidez (Araújo, Pereira \& Kac, 2007).

Quando a ansiedade materna é excessiva, pode influenciar o curso da gestação, predispondo a complicações obstétricas, como a pré-eclâmpsia (Alder, Fink, Bitzer, Hosli \& Holzgreve, 2007), o desfecho do parto, em especial, promovendo a ocorrência de parto prematuro (Dole et al., 2003; Halbreich, 2005; Hosseini et al., 2009; Meijssen, Wolf, Koldewijn, Van Baar, \& Kok, 2011), além de ser um forte preditivo também para a depressão pós-parto (Austin, Tully, \& Parker, 2007; Coelho, Murray, Royal-Lawson, \& Cooper, 2011). Nos casos em que a ansiedade se mostra exacerbada, justifica-se a adoção de medidas preventivas (Kaplan et al, 1997), como intervenções com gestantes, utilizando a ioga durante o pré-natal; também terapêuticas, como a utilização do Método Canguru com prematuros recém-nascidos de baixo peso (Arnon et al., 2014; Newham, Wittkowski, Hurley, Aplin, \& Westwood, 2014)

Também no período puerperal a ansiedade pode influenciar a relação mãe-bebê, trazendo repercussões para o desenvolvimento da criança. A ansiedade materna traria dificuldades para a relação mãe-bebê, pois uma mãe ansiosa pode ter dificuldades em compreender as demandas do recém-nascido, decodificar o seu choro e as suas necessidades de amamentação, sono e afeto, o que pode comprometer o bem-estar do bebê (Beltrami, Flores, Souza, \& Moraes, 2013). Desse modo, a ansiedade pode contribuir para a diminuição da sensibilidade materna (Dennis \& Chen, 2007; Harvey, Nicol-Haerper, \& Stein, 2007; Kaitz \& Maytal, 2005) e gerar dificuldade na capacidade materna de atender e processar informações (Dennis \& Chen, 2007), o que afeta a interação com 0 bebê (De Ruiter, Brosschot, Van Emmichhoven, \& Van ljzendoorn, 2003), acarretando maior intrusividade, ou seja, uma tendência ao controle exagerado dos comportamentos do bebê (Feldman et al., 2009; Oliveira, Frizzo, \& Marin, 2000).

Considerando que a relação mãe-bebê é fundamental para o desenvolvimento da criança, e dadas as repercussões da ansiedade materna na gravidez, no período pós-natal, na relação mãe-bebê e no desenvolvimento infantil, faz-se necessário conhecer como a ansiedade materna tem sido estudada. Assim, o objetivo deste estudo foi apresentar uma revisão sistemática da literatura sobre ansiedade materna e maternidade, considerando o período de janeiro/2010 a dezembro/2014. Com isso, buscou-se identificar e analisar a produção científica internacional e nacional existente sobre essa temática, buscando comparar as publicações de língua portuguesa e estrangeira, a fim de guiar futuras investigações.

\section{MÉTODO}

Para atingir os objetivos do estudo, foram utilizados os seguintes descritores e operadores booleanos: "ansiedade materna", "ansiedade materna" AND "maternidade" e os seus equivalentes em inglês ("maternal anxiety", "maternal anxiety" AND "motherhood") e espanhol ("ansiedad materna", "ansiedad materna" AND "maternidad"), para consultas nas seguintes bases de dados: American Search Premier Complete, Literatura Latino-Americana em Ciências da Saúde (LILACS), MEDLINE, PsycINFO, Scientific Eletronic Library Online (SciELO), e Web of Science.

Os critérios de inclusão empregados para a seleção dos itens de análise foram: a) artigos completos (empíricos ou de revisão de literatura); b) publicados entre janeiro/2009 e dezembro/2014; c) em língua portuguesa, espanhola ou inglesa; d) por autores da Psicologia, Psiquiatria e outras áreas da saúde; e) estudos empíricos cujos participantes eram mães e crianças de até três anos; e f) com foco na investigação da ansiedade da mãe (incluindo a relação entre maternidade e transtorno de ansiedade generalizada - TAG - e intervenções clínicas voltadas para essa problemática).

Já os critérios de exclusão aplicados para a seleção dos itens de análise foram: a) registros duplicados; b) que não se referiam a artigos (teses, dissertações, trabalhos de conclusão de curso, anais, livros, capítulos de livros e resenhas); c) artigos publicados fora do período considerado; d) em outros idiomas que não português, espanhol ou inglês; e) que tratavam da ansiedade da criança ou cujos participantes eram crianças maiores de três anos; f) não disponibilizados on-line, na íntegra ou gratuitamente; e g) que não contemplavam o tema de interesse, isto é, que não abordavam a ansiedade materna, que investigavam outros sintomas e/ou quadros psicopatológicos relacionados à ansiedade (por ex., transtorno de ansiedade social, transtorno de ansiedade de separação, fobia específica, transtorno do pânico, agorafobia, transtorno de ansiedade induzido por substância/medicamento, etc.), bem como que abordavam 


\section{INTERACÃO EM LF PSICOLOGIA}

exclusivamente a depressão, o retardo mental e outros quadros severos de psicopatologia (esquizofrenia, transtorno de personalidade borderline ou antissocial, regressão psicótica, risco de suicídio e abuso de substâncias químicas), além de terem como participantes os pais.

o material selecionado foi então lido na íntegra e analisado a partir das seguintes categorias: 1) ano da publicação, 2) idioma, 3) área da publicação, 4) delineamento, 5) participantes, 6) instrumentos, 7) objetivos dos estudos e 8) principais resultados e conclusões. Destaca-se que, para essa revisão, foram utilizados os principais itens de verificação de qualidade do texto de uma revisão sistemática segundo o protocolo PRISMA (Preferred Reporting Items for Systematic Reviews and Meta-Analysis) (Padula et al., 2012).
Mariana Reichelt Chemello, Daniela Centenaro Levandowski e Tagma Marina Schneider Donelli

\section{RESULTADOS E DISCUSSÃO}

$\mathrm{Na}$ busca realizada nas bases de dados, foram encontrados 2.665 registros. Foram feitas exclusões pelo período de publicação $(n=1633)$, por duplicidade de registro $(n=244)$, por formato $(n=77)$ e idioma $(n=14)$. Também foram feitas exclusões por temática (ansiedade da criança e idade dos participantes $n=357)$, por acesso restrito $(n=93)$ e outras temáticas $(n=204)$. Após a aplicação dos critérios de inclusão e exclusão, restaram 43 artigos para análise, como é mostrado na Figura 1, que sintetiza o fluxo de busca e de seleção do material.

Os 43 itens obtidos foram analisados a partir das seguintes categorias: 1) ano da publicação, 2) idioma, 3) área

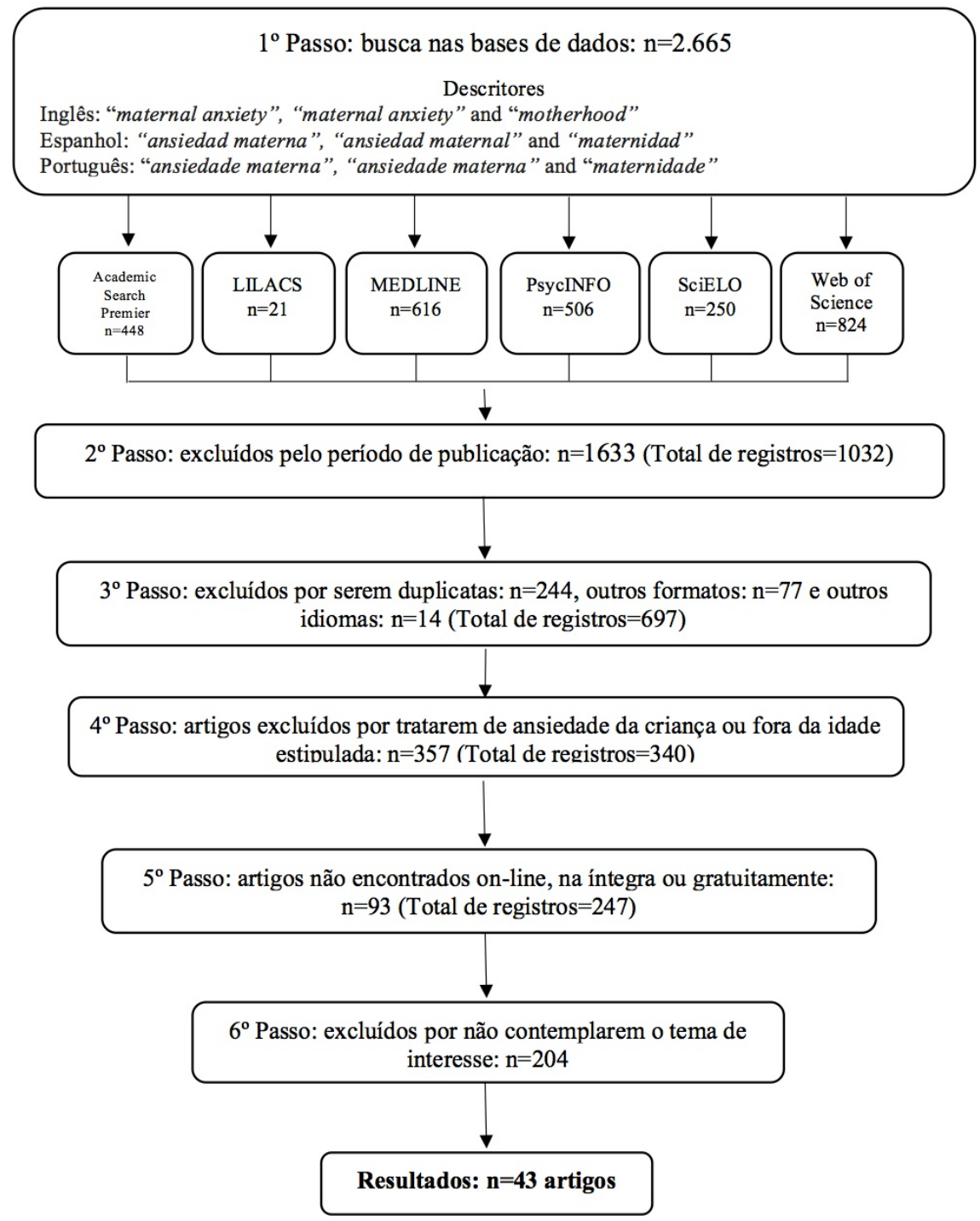

Figura 1. Fluxograma de busca e seleção do material para análise. 


\section{-4: INTERACÃO EM L* PSICOLOGIA}

da publicação, 4) delineamento, 5) participantes, 6) instrumentos, 7) objetivos dos estudos e 8) principais resultados e conclusões. As análises dessas categorias foram realizadas inicialmente de forma quantitativa, sendo posteriormente descritas qualitativamente.

\section{CATEGORIAS ANO DE PUBLICAÇÃO E IDIOMA}

0 ano em que mais ocorreram publicações de artigos em língua inglesa e espanhola foi $2014(n=11)$, ao contrário de 2012, ano em que foram localizadas menos publicações ( $n=$ 01). Nos demais anos, o número de publicações variou de oito (2013) a três (em 2010 e 2011). Considerando o idioma de publicação, foram localizados mais artigos em inglês e espanhol $(n=30)$ em comparação com os artigos publicados em português $(n=13)$. Já ao se analisar conjuntamente idioma e ano de publicação (Figura 2), verifica-se que os artigos publicados em português foram mais frequentes em 2013 e 2009 ( $n=4)$, enquanto que os artigos em inglês e espanhol o foram em $2014(n=13)$. Esses achados poderiam evidenciar uma menor produção científica brasileira sobre a temática da ansiedade materna, levando-se em conta as buscas realizadas. Contudo, entre os artigos publicados em inglês e espanhol, foram encontrados três artigos nacionais, o que pode apontar para a internacionalização da produção brasileira, visando a uma maior visibilidade na comunidade científica. A figura 2 ilustra os artigos por ano e idioma de publicação.

\section{Mariana Reichelt Chemello, Daniela Centenaro Levandowski e} Tagma Marina Schneider Donelli

\section{CATEGORIA ÁREA DA PUBLICAÇÃO}

Relativamente à área do estudo, entre as publicações em inglês e espanhol, cinco artigos foram escritos por profissionais da Psicologia (Eutrope et al., 2014; Henrichs et al., 2009; Linhares, Padovani, Carvalho, \& Martinez, 2009; Martini et al., 2013; Perosa, Ribeiro, \& Padovani, 2014), enquanto que 11 artigos em língua portuguesa foram escritos por profissionais dessa área (Airosa \& Silva, 2013; Benute et al., 2009; Chiodelli, Pereira, Rodrigues, Oliveira, \& Silva, 2014; Favaro, Peres, \& Santos, 2012; Felipe, Souza, \& Carvalho, 2014; Moraes, Beltrami, \& Souza, 2013; Padovani, Linhares, Carvalho, Duarte, \& Martinez, 2009; Perrone \& Oliveira, 2011; Ribeiro, Perosa, \& Padovani, 2014, Schmidt \& Argimon, 2009; Silveira, Padovani, Perosa, Canavez, \& Peraçoli, 2009). O maior número de publicações em língua portuguesa na área da Psicologia evidencia o interesse da área sobre o tema no Brasil.

Foram encontrados seis artigos em língua inglesa envolvendo Psicologia e Psiquiatria (Arteche et al., 2011; Beebe, Steele, Jaffe, \& Stehle, 2011; Dennis, Coghlan, \& Vigod, 2013; O'Connor, Monk, \& Fitelson, 2014; Reck, Noe, \& Gerstenlauer, 2012; Richter \& Reck, 2013); dois em língua portuguesa envolvendo Psicologia e Fonoaudiologia (Beltrami et al., 2013; Dias, Beltrami, \& Souza, 2013); um artigo em língua inglesa em conjunto entre Psicologia e Pediatria (Newham et al., 2014); um outro em língua inglesa englobando Psicologia, Psiquiatria, Ginecologia e Obstetrícia (Sockol, Epperson, \& Barber, 2014) e um em língua inglesa que reuniu pesquisadores da Psicologia, Psiquiatria e Neurologia (Consonni et al., 2010).

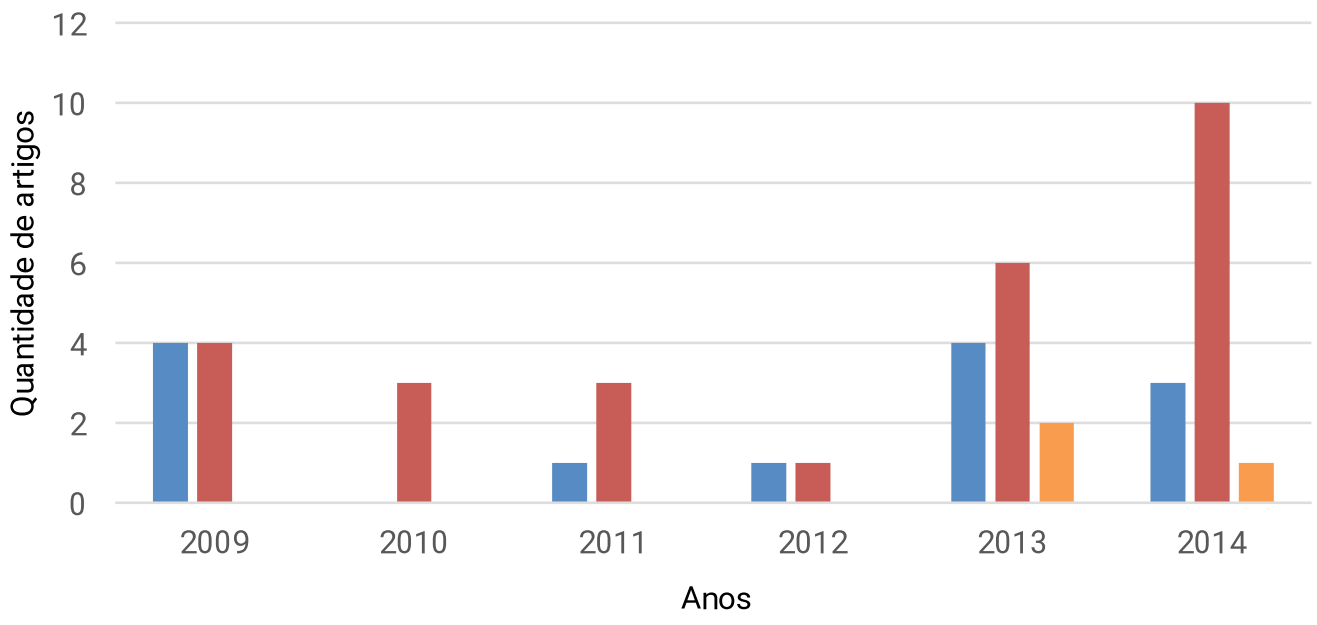

- Língua Portuguesa Língua Inglesa Língua Espanhola

Figura 2. Distribuição dos artigos por ano e por idioma de publicação 


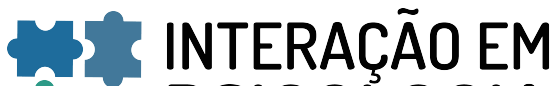 ET PSICOLOGIA}

Mariana Reichelt Chemello, Daniela Centenaro Levandowski e Tagma Marina Schneider Donelli

Nas demais áreas da saúde, destacou-se Ginecologia e Obstetrícia, com quatro artigos publicados, sendo três em língua inglesa (Giakoumaki, Vasilaki, Lili, Skouroliakou, \& Liosis, 2009; Glover, 2014; Liou, Wang, \& Cheg, 2014) e um em língua espanhola (Romero-Gutiérrez, Rocha-Morales, \& RuizTreviño, 2013); seguido pela Enfermagem ( $n=3)$, sendo um em língua espanhola (Elizarraraz, Argote, Frausto, \& Toscano, 2014) e dois em língua inglesa (Erdem, 2009; Sherestha, Adachi, Petrini, \& Sherestha, 2014). Em Pediatria foram encontrados três artigos ( $n=3)$, dois em língua inglesa (Arnon et al., 2014; Pérez, Bellót, Pérez \& Herrera, 2013) e um em língua espanhola (Zelkowitz, Na, Wang, Bardin, \& Papageorgiou, 2011). Em Saúde Pública foi localizado apenas um artigo $(n=1)$ em língua inglesa (Nasreen, Kabir, Forsell, \& Edhborg, 2010).

Entre as publicações conjuntas na área da saúde, excluindo-se a Psicologia, foi encontrado um artigo nas áreas de Psiquiatria, Neurologia, Ginecologia e Obstetrícia (Qiu et al., 2013); um de Pediatria, Ginecologia e Obstetrícia (Paul, Downs, Schaefer, Beiler, \& Weisman, 2013); um de Ginecologia, Obstetrícia e Saúde Pública (Sanchez et al, 2013); e um de Ginecologia, Obstetrícia e Epidemiologia (Catov, Abatemarco, Markovic, \& Roberts, 2010), todos em língua inglesa. Esses achados indicam que a temática da ansiedade materna, embora seja predominantemente estudada pela Psicologia, também é foco de interesse de profissionais de outras áreas da saúde, cujo trabalho envolve a assistência a gestantes, puérperas e/ou mães e seus bebês.

\section{CATEGORIA DELINEAMENTO}

Quanto ao delineamento dos estudos, dos 30 artigos encontrados em língua inglesa ou espanhola, 27 eram artigos empíricos quantitativos (Arnon et al., 2014; Arteche et al., 2011; Beebe et al., 2011; Catov et al., 2010; Consonni et al., 2010, Dennis et al., 2013; Elizarraraz et al., 2014; Erdem, 2009; Eutrope et al., 2014; Giakoumaki et al., 2009; Henrichs et al., 2009; Linhares et al., 2009; Liou et al., 2014; Martini et al., 2013; Nasreen et al., 2010; Newham, et al., 2014; Paul et al., 2013; Pérez et al., 2013; Perosa et al., 2014; Qiu et al., 2013; Reck et al., 2012; Richter \& Reck, 2013; Romero-Gutiérrez al., 2013; Sanchez et al., 2013). Apenas três artigos quantitativos tinham caráter longitudinal (Sherestha et al., 2014; Sockol et al., 2014; Zelkowitz et al., 2011). Outros três artigos tinham caráter qualitativo do tipo revisão de literatura (Glover, 2014; O'Connor et al., 2014; Rallis, Skouteris, McCabe, \& Milgrom, 2014)

Já em língua portuguesa, 10 estudos quantitativos de caráter empírico foram localizados (Airosa \& Silva, 2013; Beltrami et al., 2013; Benute et al., 2009; Chiodelli et al., 2014;
Favaro et al., 2012; Moraes et al., 2013; Padovani et al., 2009; Ribeiro et al., 2014; Schmidt \& Argimon, 2009; Silveira et al., 2009) e três qualitativos, organizados como estudos de caso (Dias et al., 2013; Felipe et al., 2014; Perrone \& Oliveira 2011). Observa-se o predomínio de artigos quantitativos na maioria das publicações, tanto nas publicações estrangeiras (27 artigos em inglês e espanhol), quanto nas publicações em língua portuguesa (10 artigos). A figura 3 ilustra a distribuição dos dados por idioma de publicação e por origem dos dados utilizados.

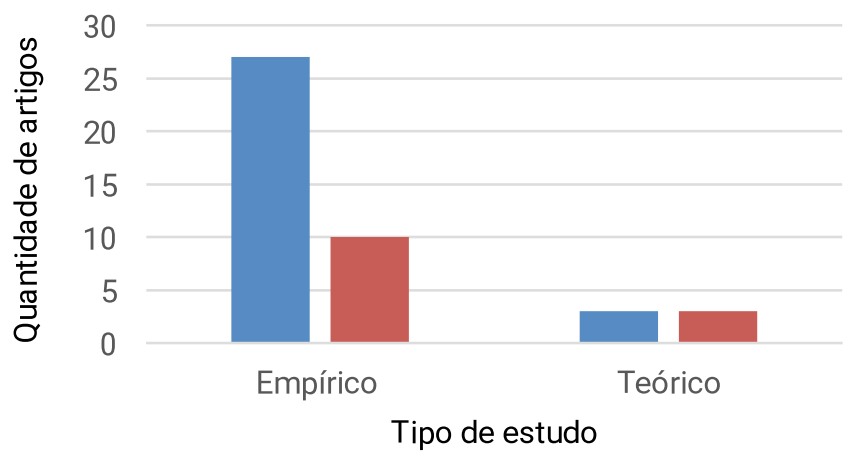

$$
\text { - Língua Estrangeira " Língua Portuguesa }
$$

Figura 3. Distribuição dos artigos por idioma de publicação e por origem dos dados

\section{CATEGORIA PARTICIPANTES}

Com relação aos participantes dos estudos empíricos publicados em língua espanhola e inglesa, foram identificados: 1) gestantes $(n=8)$ (Catov et al., 2010; Consonni et al., 2010; Elizarraraz et al., 2014; Linhares et al., 2009; Martini et al., 2013; Newham et al., 2014; Reck et al., 2012; Sherestha et al., 2014), 2) mães e/ou puérperas ( $n=12)$ (Arteche et al., 2011; Dennis et al., 2013; Erdem, 2009; Giakoumaki et al., 2009; Henrichs et al., 2009; Liou et al., 2014; Nasreen et al., 2010; Qiu et al., 2013; Romero-Gutiérrez et al., 2013; Sanchez et al., 2013; Sherestha et al., 2014; Sockol et al., 2014) e 3) díades mãe-bebê ( $n=8)$ (Arnon et al., 2014; Beebe et al., 2011; Eutrope et al., 2014; Paul et al., 2013; Pérez et al., 2013; Perosa et al., 2014; Richter \& Reck, 2013; Zelkowitz et al., 2011). A quantidade de participantes por estudo teve grande variação, tendo sido encontrados desde 40 até mais de 2.000 .

Em relação a esse aspecto, nas publicações em língua portuguesa foram identificados: 1) estudos com gestantes (n = 1) (Schmidt \& Argimon, 2009), 2) com gestantes e puérperas ( $n=1$; Airosa \& Silva, 2013), 3) com mães e/ou puérperas $(n=6)$ (Benute et al., 2009; Favaro et al., 2012; 
Felipe et al., 2014; Padovani et al., 2009; Perrone \& Oliveira, 2011; Silveira et al., 2009), e 4) com díades mãe-bebê ( $n=5)$ (Beltrami et al., 2013; Chiodelli et al., 2014; Dias et al., 2013; Moraes et al., 2013; Ribeiro et al., 2014). O número de participantes por estudo teve variação de oito a 182 participantes.

Os resultados referentes aos participantes apontam que, dos 43 estudos analisados, 11 tiveram como foco apenas as mães (cinco estudos internacionais e seis estudos nacionais) e 13 as díades mãe-bebê, o que está em acordo ao tema dessa revisão. Entretanto, embora gestantes e puérperas muito frequentemente tenham sido alvo dos estudos internacionais, destacou-se a escassez de estudos com esse público entre aqueles publicados em português no período considerado. Esse achado indica uma lacuna da produção científica que merece ser preenchida, devido à importância da detecção da ansiedade materna pelos profissionais já na gestação, devido aos seus eventuais impactos no percurso da gravidez e na vivência do parto.

\section{CATEGORIA INSTRUMENTOS}

Com relação aos instrumentos empregados, a maioria dos estudos de língua inglesa e espanhola utilizou o StateTrait Anxiety Inventory (STAI) para avaliar a ansiedade materna ( $n=16$ ) (Arnon et al., 2014; Beebe et al., 2011; Catov et al., 2010; Consonni et al., 2010; Dennis et al., 2013; Elizarraraz et al., 2014; Erdem, 2009, Giakoumaki et al., 2009; Linhares et al., 2009; Nasreen et al, 2010; Newham et al., 2014; Paul et al, 2013; Pérez et al., 2013; Qiu et al., 2013; Richter \& Reck, 2013; Zelkowitz et al., 2011). Esse último artigo também utilizou o Wijma Delivery Expectancy Questionnaire (WDEQ) para avaliar o medo do parto como indicador de ansiedade específica da gravidez. Já nos estudos publicados em português, três (Chiodelli et al., 2014; Padovani et al., 2009, Silveira et al., 2009) também utilizaram instrumento equivalente ao STAI, ou seja, o IDATE (Inventário de Ansiedade Traço-Estado). Ainda, foram encontrados quatro artigos (Beltrami et al., 2013; Dias et al., 2013; Moraes et al., 2013, Schmidt \& Argimon, 2009) que utilizaram o BAI (Beck Anxiety Inventory).

Interessante destacar que, para além da ansiedade, alguns estudos utilizaram instrumentos para avaliar diferentes estados e atitudes em relação à maternidade e comportamentos do bebê, como por exemplo, Attitudes Toward Motherhood Scale (AToM), Dysfunctional Attitudes Scale (DAS), e The Maternal Attitudes Questionnaire (MAQ) (Sockol et al., 2014). Já quanto ao comportamento do bebê, verificou-se o uso da Neonatal Behavioural Assessment Scale (NBAS) em Glover (2014).

\section{CATEGORIA OBJETIVOS DOS ESTUDOS}

Quanto aos objetivos dos estudos, foi possível agrupá-los em três grandes eixos temáticos: 1) artigos que avaliaram a ansiedade em gestantes, puérperas e mães $(n=14$ publicações), 2) artigos que abordaram, além da ansiedade, outros sintomas e/ou quadros psicopatológicos, tais como depressão e estresse ( $n=23)$, e 3) estudos sobre intervenções voltadas para o manejo da ansiedade $(n=6)$.

Os artigos do primeiro eixo relacionaram a ansiedade (seja da gestante, puérpera ou mãe) com o desenvolvimento infantil (Padovani et al., 2009; Perosa et al., 2014; Ribeiro et al., 2014; Richter \& Reck, 2013), com a internação em Unidade de Tratamento Intensivo (UTI) neonatal e complicações fetais (Erdem, 2009; Qiu et al., 2013; Romero-Gutiérrez et al., 2013), com a comunicação e a interação mãe-bebê (Beebe et al., 2011; Dias et al., 2013; Henrichs et al., 2009), com variáveis sociodemográficas no período do pós-parto (Chiodelli et al., 2014; Dennis et al., 2013; Sherestha et al., 2014) e a relação entre ansiedade materna, otimismo, idade gestacional e peso ao nascer (Catov et al., 2010).

Já os artigos do segundo eixo abordaram, além da ansiedade, outros sintomas e/ou quadros psicopatológicos, tais como depressão e estresse, e pesquisaram a sua relação com o desenvolvimento infantil (Beltrami et al., 2013; Giakoumaki et al., 2009; Glover, 2014; Liou et al., 2014; Moraes et al., 2013; Nasreen et al, 2010; O'Connor et al., 2014; Paul et al., 2013; Silveira et al., 2009; Sockol et al., 2014), amamentação (Pérez et al., 2013), risco de parto prematuro (Elizarrarz et al., 2014; Eutrope et al., 2014; Sanchez et al, 2013), aborto (Benute et al., 2009), suporte social, apego e vínculo (Airosa \& Silva, 2013; Schmidt \& Argimon, 2009), autoconfiança materna (Reck et al., 2012) e processamento de expressões faciais dos bebês (Arteche et al., 2011).

Alguns estudos desse segundo eixo apenas verificaram a relação entre ansiedade e estresse (Rallis et al., 2014) e entre transtornos de ansiedade (na gravidez e no pós-parto) e sua comorbidade com transtornos depressivos (Martini et al., 2013). Ansiedade e depressão também foram comparadas entre mães de bebês prematuros e a termo em dois estudos (Favaro et al., 2012; Felipe et al., 2014).

Por fim, dentre os estudos do terceiro eixo, que trataram de intervenções voltadas para o manejo da ansiedade, encontrou-se a investigação dos benefícios para o bebê prematuro e suas mães do canto materno no método canguru (Arnon et al., 2014) e da ioga para reduzir ansiedade durante a gravidez (Newham et al., 2014). Outros estudos avaliaram a ansiedade e propuseram intervenções como o programa multidisciplinar de preparação para o parto e maternidade (Consonni et al., 2010), e intervenções lúdicas 


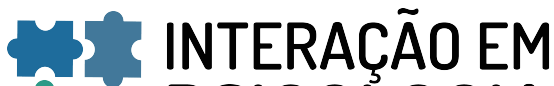 ET PSICOLOGIA}

Mariana Reichelt Chemello, Daniela Centenaro Levandowski e Tagma Marina Schneider Donelli grupais no controle da ansiedade com filmagem da interação mãe-bebê durante o jogo (Perrone \& Oliveira, 2011); e analisaram os efeitos da ansiedade em mães sugerindo programas de avaliação precoce em mães com bebês de muito baixo peso (Zelkowitz et al., 2011). Outro estudo buscou avaliar e comparar sintomas de ansiedade e depressão antes e depois de intervenção psicológica com mães de bebês pré-termo com muito baixo peso internados em UTI Neonatal (Linhares et al., 2009).

Observa-se diversidade dos artigos pesquisados quanto ao estudo da ansiedade materna (identificação, avaliação e/ou intervenção) no terceiro eixo. Destaca-se a escassez de estudos relacionados a intervenções voltadas para o manejo da ansiedade, especialmente de cunho preventivo. Assim, embora se busque a avaliação da ansiedade materna, pouco ainda tem sido feito (ou pelo menos divulgado) acerca da assistência prestada às mulheres no ciclo gravídicopuerperal. Nesse sentido, percebe-se a carência de pesquisas que realizem um acompanhamento sistemático da mãe desde o pré-natal até o pós-parto. A figura 4 ilustra a distribuição dos artigos por objetivos dos estudos.

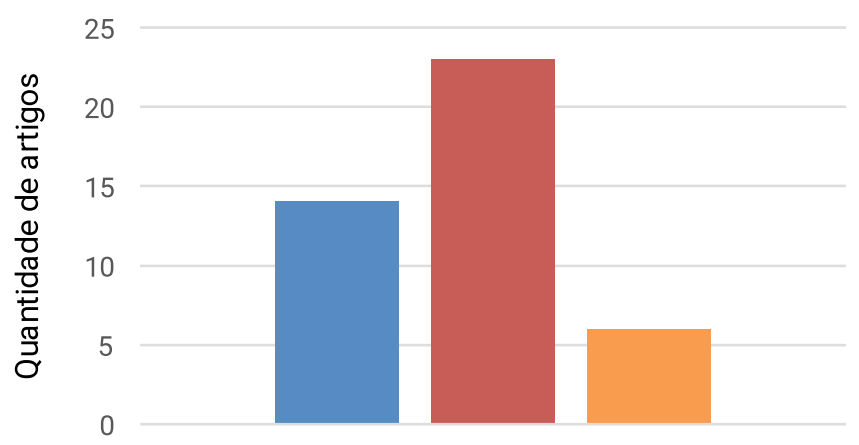

- Ansiedade em gestantes, mães e puérperas

- Ansiedade e outros sintomas como depressão e estresse

घstudos sobre intervenção para manejo ansiedade

Figura 4. Distribuição dos artigos por objetivos dos estudos

\section{CATEGORIA PRINCIPAIS RESULTADOS E CONCLUSÕES}

No que diz respeito aos principais resultados dos estudos, esses foram agrupados em seis eixos temáticos, considerando a relação entre: 1) ansiedade materna, internação em UTI neonatal, complicações fetais e risco de parto prematuro; 2) ansiedade materna, variáveis sociodemográficas e desenvolvimento infantil; 3) ansiedade materna e outros sintomas e/ou quadros psicopatológicos, tais como depressão e estresse, e desenvolvimento infantil; 4) ansiedade materna, comunicação e relação mãe-bebê; 5) ansiedade materna, relação conjugal e apoio social; e, por fim, 6) resultados de intervenções voltadas para o manejo da ansiedade.

Os estudos do primeiro eixo (ansiedade materna, internação em UTI neonatal, complicações fetais e risco de parto prematuro) evidenciaram relação entre o tempo de internação do bebê em UTI Neonatal e aumento da ansiedade das mães, além de ter apontado a ansiedade como fator que dificulta a capacidade materna de responder sensivelmente aos sinais do bebê (Erdem, 2009; Linhares et al., 2009; Zelkowitz et al., 2011). A ansiedade materna aumentada também esteve relacionada a complicações obstétricas e fetais, como ameaça de aborto, risco de parto prematuro e baixo peso ao nascer (Romero-Gutiérrez et al., 2013). Também foi apontada a maior incidência de ameaça de aborto e parto prematuro (Benute et al., 2009) em mulheres com índices elevados de ansiedade. A presença de sepse neonatal e taquipnéia transitória do recém-nascido, bem como o desenvolvimento mais lento do hipocampo, também foram observados em filhos de mães ansiosas (Qiu et al., 2013).

Outros estudos foram alocados no segundo eixo, denominado "ansiedade materna, variáveis sociodemográficas e desenvolvimento infantil”. Eles indicaram variáveis sociodemográficas que influenciam a ansiedade materna e também se encontram associadas ao desenvolvimento do bebê (Catov et al., 2010; Chiodelli et al., 2014; Moraes et al., 2013; Padovani et al., 2009; Perosa et al., 2014; Ribeiro et al., 2014; Richter \& Reck, 2013; Sherestha et al., 2014). Entre essas variáveis, podem ser citadas: a escolaridade materna (menores índices), nível socioeconômico (baixo), idade materna (menor idade) e o desconhecimento acerca de cuidados com o recém-nascido. Particularmente, os estudos desse grupo consideraram os atrasos no desenvolvimento infantil e os riscos ao desenvolvimento motor amplo decorrentes da ansiedade materna.

Estudos agrupados no terceiro eixo (ansiedade materna e outros sintomas e/ou quadros psicopatológicos, tais como depressão e estresse, e desenvolvimento infantil) demonstraram haver relação entre ansiedade materna e outros sintomas e/ou quadros psicopatológicos, tais como depressão e estresse. Alguns estudos (Beltrami et al., 2013; Glover, 2014; Moraes et al., 2013; Nasreen et al, 2010; O'Connor et al., 2014) identificaram associação entre a presença de ansiedade materna e de depressão, relacionada com baixo peso ao nascer, risco de parto prematuro e prejuízos no desenvolvimento infantil, tais como formação de apego inseguro e risco de prejuízos na aquisição da linguagem, devido a dificuldades no diálogo e na interação 


\section{H. INTERACÃO EM K PSICOLOGIA}

Mariana Reichelt Chemello, Daniela Centenaro Levandowski e Tagma Marina Schneider Donelli mãe-bebê. Outro estudo constatou maior incidência de sintomas clinicamente significativos de ansiedade e depressão entre mães de bebês prematuros $(75 \%$ e $50 \%$, respectivamente), em comparação a mães de bebês a termo, pois destas, $65 \%$ não apresentavam sintomas clinicamente significativos de ansiedade e depressão. Assim, verifica-se como o nascimento prematuro pode ser traumático para as mães e interferir na saúde mental materna (Favaro et al., 2012; Felipe et al., 2014). Em outro estudo, ansiedade, depressão e estresse mostraram-se relacionados (Rallis et al., 2014) e influenciaram a amamentação, não apenas na redução da produção de leite, como também na interação mãe-bebê durante o ato de amamentar (Pérez et al., 2013). Também foi verificada uma relação entre a ansiedade, depressão e estresse e risco de parto prematuro (Eutrope et al., 2014; Sanchez et al, 2013). Reck et al. (2012) demonstraram ainda uma ligação significativa entre transtornos de ansiedade e depressão e prejuízos na autoconfiança materna. Por fim, Martini et al. (2013) concluíram que transtornos de ansiedade são fatores de risco para sintomas depressivos e que transtornos depressivos, em comorbidade, podem agravar os transtornos de ansiedade.

Artigos do quarto eixo, denominado "ansiedade materna, comunicação e relação mãe-bebê", indicaram que a ansiedade materna afeta a comunicação e o diálogo mãebebê, por influenciar negativamente a capacidade de resposta materna (Arteche et al., 2011; Beebe et al., 2011; Dias et al., 2013; Henrichs et al., 2009). Arteche et al. (2011) concluíram que mães com transtornos de ansiedade tendiam a identificar expressões faciais positivas em menor intensidade, sugerindo dificuldades no processamento das expressões faciais, podendo levar a uma menor capacidade de resposta materna e comprometer a qualidade de interação mãe-bebê.

Artigos do quinto eixo (ansiedade materna, relação conjugal e apoio social) identificaram a relação entre ansiedade materna e apoio social. O suporte social mostrouse negativamente relacionado com a ansiedade (Airosa \& Silva, 2013). Mulheres com relacionamento conjugal (dificuldades conjugais) e apoio social (insuficiente) estão mais vulneráveis ao desenvolvimento de ansiedade severa (Sherestha et al., 2014).

Já no último eixo, denominado "resultados de intervenções voltadas para o manejo da ansiedade", estudos demonstraram que intervenções voltadas para o manejo da ansiedade de gestantes apresentaram resultados positivos, favorecendo às mães assumir sua maternidade com menos ansiedade (Consonni et al., 2010; Perrone \& Oliveira, 2011; Zelkowitz et al., 2011). Da mesma forma, a ioga nesse período contribuiu para a redução da ansiedade antes do parto e preveniu a intensificação da sintomatologia depressiva (Newham et al., 2014). Já no pós-parto, foi observada redução dos níveis de ansiedade e depressão após uma intervenção psicológica com mães de bebês prétermo com muito baixo peso internados em UTI (Linhares et al., 2009), confirmando a necessidade de apoio psicológico a essas mães. Outro estudo (Arnon et al., 2014) mostrou que o cantar materno durante a aplicação do Método Canguru reduziu a ansiedade materna e proporcionou estabilidade aos bebês prematuros.

\section{CONSIDERAÇÕES FINAIS}

A partir desta revisão da literatura sobre ansiedade materna e maternidade, considerando artigos científicos publicados entre janeiro/2009 e dezembro/2014, foi verificado que o tema vem sendo estudado por pesquisadores de diferentes países e várias áreas de formação dentro da Saúde, especialmente nos últimos anos. Esse achado surpreende, tendo em vista que a ênfase maior é costumeiramente dada à depressão materna, considerada umas das principais complicações da vivência da maternidade. Entretanto, como ansiedade e depressão costumam estar associadas, é importante um olhar também para a identificação e o manejo da ansiedade, o que já tem sido considerado por vários pesquisadores.

De fato, após a análise dos estudos revisados, destaca-se a importância de identificar e avaliar a ansiedade já na gestação, como medida preventiva e protetiva para a saúde mental das mães, o desenvolvimento do bebê e a qualidade da relação dessa díade. Percebeu-se especialmente na área de Ginecologia e Obstetrícia um interesse nesse diagnóstico precoce, buscando uma melhor assistência à mulher.

Contudo, muito embora haja necessidade de detecção precoce da ansiedade, percebe-se a ausência de um instrumento específico para avaliar a ansiedade materna. Os instrumentos mais frequentemente empregados para essa avaliação nos estudos revisados (STAl e BAI) não consideram as questões típicas da maternidade, que podem provocar o seu aparecimento e intensificação, tais como as preocupações que comumente são encontradas nas mulheres nesse período, relativas à saúde do bebê e à própria saúde, bem como às mudanças de vida decorrentes da maternidade. Da mesma forma, também o estresse destacou-se como um tópico importante no contexto da maternidade e no estudo da ansiedade materna. Assim, verifica-se a necessidade de aprofundar estudos relacionando ansiedade e estresse maternos, devido a características peculiares à gestação e à maternidade, que 


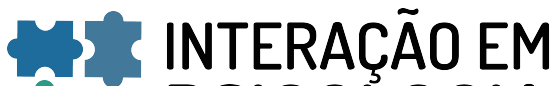 ET. PSICOLOGIA}

Mariana Reichelt Chemello, Daniela Centenaro Levandowski e Tagma Marina Schneider Donelli

podem desencadear esses estados emocionais.

Observou-se o predomínio de artigos empíricos quantitativos, tanto internacionais como nacionais. Apenas três artigos internacionais possuíram caráter longitudinal, ao passo que nenhum artigo nacional realizou estudo longitudinal. Neste sentido, evidencia-se a falta de estudos longitudinais que proporcionem 0 acompanhamento sistemático da mãe desde a gestação até o puerpério, dada a necessidade da prevenção e promoção da saúde mental materna. Tal delineamento permitiria conhecer as especificidades do fenômeno da ansiedade materna ao longo do tempo, possibilitando entender seu desenvolvimento, mudanças e implicações para a saúde da mulher e para o desenvolvimento do bebê.

Percebe-se, portanto, a necessidade de aprofundamento das investigações na área, devido às implicações do estado emocional ansioso na vivência da gravidez, da maternidade, na relação mãe-bebê e no próprio desenvolvimento fetal. A partir dos estudos revisados, ficou evidente que a ansiedade no contexto da maternidade é um fenômeno multifatorial, relacionado a diferentes variáveis, tanto sociodemográficas (por ex., idade, escolaridade, nível socioeconômico) como psicossociais (ex., tipo de apego, suporte social). Tais variáveis tanto podem desencadear um estado ansioso na mãe, como podem ser influenciadas pela ansiedade daquelas mães que já a possuem como uma característica de sua personalidade. Essa complexidade exige do profissional um olhar individualizado para cada mulher, em busca de um entendimento maior sobre a origem da ansiedade materna frente aos aspectos típicos da maternidade.

Em síntese, este estudo propiciou a identificação do panorama da produção científica recente sobre uma temática complexa e ainda aberta a novas investigações. Salienta-se a importância de abarcar, nas futuras pesquisas, para além da construção de instrumentos específicos de avaliação da ansiedade materna, adaptados à realidade brasileira, a eficácia e a efetividade de diferentes intervenções e tratamentos para o manejo dessa condição, que tem efeitos adversos não apenas para as mulheres, mas também para seus bebês e suas famílias.

\section{CONTRIBUIÇÃO DE CADA AUTOR}

M.R.C. realizou a análise formal dos dados, a conceitualização, a investigação, a tabulação dos dados e preparou o rascunho original; M.R.C., D.C.L. e T.M.S.D. foram responsáveis pela metodologia e a visualização; D.C.L. e T.M.S.D. administraram o projeto, supervisionaram, validaram os dados, revisaram e editaram a redação.

\section{DECLARAÇÃO DE CONFLITOS DE INTERESSE}

Os autores declaram que não há conflitos de interesses no presente artigo.

\section{REFERÊNCIAS}

Alder, J., Fink, N., Bitzer, J., Hosli, I., \& Holzgreve, W. (2007). Depression and anxiety during pregnancy: a risk factor for obstetric, fetal and neonatal outcome? A critical review of the literature. Journal of Maternal-fetal Medicine Neonatal, 20(3), 189-209.

Airosa, S., \& Silva, I. (2013). Associação entre vinculação, ansiedade, depressão, estresse e suporte social na maternidade. Psicologia, Saúde \& Doenças, 14(1), 64-77.

Araújo, D. M. R, Pereira, N. L., \& Kac, G. (2007). Ansiedade na gestação, prematuridade e baixo peso ao nascer: Uma revisão sistemática da literatura. Cadernos Saúde Pública, 23(4), 747-756.

Arnon, S., Diamant, C., Bauer, S., Regev, R., Sirota, G., \& Litmanovitz, I. (2014). Maternal singing during kangaroo care led to autonomic stability in preterm infants and reduced maternal anxiety. Acta Pediatrica, 103, 1039-1044. http://dx.doi.org/10.1111/apa.12744

Arteche, A., Joormann, J., Harvey, A., Craske, M. C., Gotlib, I. H., Lehtonen, A., Counsell, N., \& Stein, A. (2011). The effects of postnatal maternal depression and anxiety on the processing of infant faces. Journal of Affective Disorders, 172 , 324-330. htto://dx.doi.org/10.1016/j.jad.2011.04.015

Austin, M. P., Tully, L., \& Parker, G. (2007). Examining the relationship between antenatal anxiety and postnatal depression. Journal of Affective Disorders, 101(1-3), 169174. https://doi.org/10.1016/j.jad.2006.11.015

Barbirato, F., \& Dias, G. (2009). A mente do seu filho: Como estimular crianças e identificar distúrbios psicológicos na infância. Rio de Janeiro: Agir.

Beebe, B., Steele, M., Jaffe, J., \& Stehle, E. (2011). Maternal anxiety symptons and mother-infant self -and interactive contingency. Infant Mental Health Journal, 32(2), 174-206. http://dx.doi.org/10.1002/imhj.20274

Beltrami, L., Flores, M. R., Souza, A. P. R. S., \& Moraes, A. B. (2013). Associação entre indicadores de risco ao desenvolvimento e estado emocional materno. Revista CEFAC, 15(2), 348-360.

Benute, G. R. G., Nomura, R. M. Y., Pereira, P. P., Lucia, M. C. S., \& Zugaib, M. (2009). Abortamento espontâneo e provocado: ansiedade, depressão e culpa. Revista da Associação Médica Brasileira, 5(3), 322-327. http://dx.doi.org/10.1590/S0104-42302009000300027 


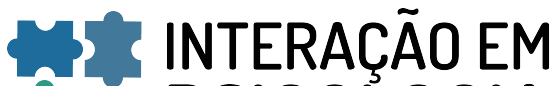 IT PSICOLOGIA}

Catov, J. M., Abatemarco, D. J., Markovic, N., \& Roberts, J. M. (2010). Anxiety and optimism associated with gestational age at birth and fetal growth. Maternal and Child Health Journal, 14, 758-764. http://dx.doi.org/10.1007/s10995009-0513-y

Chiodelli, T., Pereira, V. A., Rodrigues, O. M. P. R., Oliveira, C. S., \& Silva, V. F. M. (2014). Desenvolvimento do bebê nos dois primeiros meses de vida: Variáveis maternas e sociodemográficas. Pensando Famílias, 18(1), 64-77.

Coelho, H. F., Murray, L., Royal-Lawson, M., \& Cooper, P. J. (2011). Antenatal anxiety disorder as a predictor of postnatal depression: A longitudinal study. Journal of Affective Disorders, 129(1-3), 348-353.

Consonni, E. B., Calderon, I. M. P., Consonni, M., Conti, M. H. S., Prevedel, T. T. S., \& Rudge, M. V. C. R. (2010). A multidisciplinary program of preparation for childbirth and motherhood: Maternal anxiety and perinatal outcomes. Reproductive Health, 7 (28), 1-6.

Dennis, T. A., \& Chen, C. C. (2007). Emotional face processing and attention performance in three domains: Neurophysiological mechanisms and moderating effects of trait anxiety. International Journal of Psychophysiology, 65(1), 10-19

Dennis, C. L., Coghlan, M., \& Vigod, S. (2013). Can we identify mothers at-risk for postpartum anxiety in the immediate postpartum period using the State-Trait Anxiety Inventory? Journal Affective Disorders, 150(3), 1217-1220. http://dx.doi.org/10.1016/j.jad.2013.05.049.

De Ruiter, C., Brosschot, J., Van Emmichoven, I. A. Z., \& Van ljzendoorn, M. H. (2003). Selective processing of threatening information: Effects of attachment representation and anxiety disorder on attention and memory. Development Psychopathology, 15(1), 219-237.

Dias, L. O., Beltrami, L., \& Souza, A. P. R. (2013). Ansiedade e depressão em mães de crianças com distúrbios de linguagem: A importância do trabalho interdisciplinar. Fractal: Revista de Psicologia, 25(3), 515-530.

Dole, N., Savitz, D. A., Hertz-Picciotto I., Siega-Riz, A. M., Mcmahon, M. J., \& Buekens, P. (2003). Maternal stress and preterm birth. American Journal of Epidemiology, 157(1), 14-24.

Elizarraraz, N. L. A., Argote, G. V., Frausto, M. A. M., \& Toscano, L. M. (2014). Riesgo de trabajo de parto pretérmino espontâneo y su relación com estrès y ansiedad. Index de Enfermaría, 23(1-2), 21-25.

Erdem, Y. (2009). Anxiety levels of mothers whose infants have been cared for in unit level-I of a neonatal intensive care unit in Turkey. Journal of Clinical Nursing, 19, 17381747.

2702.2009.03115.x
Eutrope, J., Aurore, T., Lempp, F., Aupetit, L., Saad, S., Dodane, C., Bednarek, N., De Mare, L., Sibertin-Blanc, D., Nezelof, S., \& Rolland, A. C. (2014). Emotional reactions of mothers facing premature births: Study of 100 mother-infant dyads 32 gestational weeks. PLOS ONE, 9(8), 1-7. doi: 10.1371/journal.pone.0104093

Favaro, M. S. F., Peres, R. S., \& Santos, M. A. (2012). Avaliação do impacto da prematuridade na saúde mental de puérperas. Psico-USF, 17(3), 457-465.

Feldman, R., Granat, A., Pariente, C., Kanety, H., Kuint, J., \& Gilboa-Schechtman, E. (2009). Maternal depression and anxiety across the postpartum year and infant social engagement, fear regulation, and stress reactivity. Journal of American Academic of Child \& Adolescent Psychiatry, 48, 919-927.

Felipe, A. O. B., Souza, J. J., \& Carvalho, A. M. P. (2014). Impactos do nascer prematuro na saúde mental das mães. Arquivos de Ciências da Saúde, 21(3) 16-27.

Giakoumaki, O., Vasilaki, K., Lili, L., Skouroliakou, M., \& Liosis, G. (2009). The role of maternal anxiety in the early postpartum period: screening for anxiety and depressive symptomatology in Greece. Journal of Psychosomatic Obstetrics \& Gynecology, 30(1), 21-28. http://dx.doi.org/10.1080/01674820802604839

Glover, V. (2014). Maternal depression, anxiety and stress during pregnancy and child outcome: What needs to be done. Best Practice \& Research Clinical Obstetrics and Gynaecology, 28 (1), 25-35.

Gorenstein, C., Andrade, L., \& Zuardi, A.W. (Orgs.) (2000). Escalas de avaliação clínica em psiquiatria e psicofarmacologia. São Paulo: Lemos-Editorial.

Halbreich, U. (2005). The association between pregnancy processes, preterm delivery, low birth weight, and postpartum depressions: The need for interdisciplinary integration. American Journal of Obstetrics Gynecology, 193(4), 1312-1322.

Harvey, A. G., Nicol-Haerper, R., \& Stein, A. (2007). Interactions between mothers and infants: Impact of maternal anxiety. Infant Behavior Development, 30(1), 161-167.

Henrichs, J., Schenk, J. J., Schmidt, H. G., Velders, F. P., Hofman, A., Jaddoe, V. W. V., Verhulst, F. C., \& Tiemeier, H. (2009). Maternal pre- and postnatal anxiety and infant temperament: The generation $\mathrm{R}$ study. Infant and Child Development, 18(6), 556-572 doi: 10.1002/Icd.639

Hosseini, S. M., Biglan, M. W., Larkby, C., Brooks, M. M., Gorin, M. B., \& Day, N. L. (2009). Trait-anxiety in pregnant women predicts offspring birth outcomes. Paediatric Perinatal Epidemiology, 23(6), 557-566.

Kaitz, M., \& Maytal, H. (2005). Interactions between anxious mothers and their infants: An integration of theory and research findings. Infant Mental Health Journal, 26, 570597. 


\section{H MTERAC̄OAEM ET PSICOLOGIA}

Kaplan, H. I., \& Sadock, B. J. (2000). Comprehensive Textbook of Psychiatry. (7.ed.) Baltimore M D: Lippincott Williams \& Wilkins.

Kaplan, H. I., Sadock, B. J., \& Grebb, J. A. (1997). Compêndio de psiquiatria: Ciência do Comportamento e Psiquiatria Clínica. ( $7^{\mathrm{a}}$ ed). Porto Alegre: Artes Médicas.

Linhares, M. B. M., Padovani, F. H. P., Carvalho, A. E. V. C., \& Martinez, F. E. (2009). Anxiety and depression in mothers of preterm infants and psychological intervention during hospitalization in neonatal ICU. The Spanish Journal of Psychology, 12(1), 161-170.

Liou, S. R., Wang, P., \& Cheng, C. Y. (2014). Longitudinal study of perinatal maternal stress, depressive symptoms and anxiety. Midwifery, 30, 95-801.

Martini, J., Wittich, J., Petzoldt, J., Winkel, S., Einsle, F., Siegert, J., Höfler, M., Beesdo-Baum, K., \& Wittchen, H. U. (2013). Maternal anxiety disorders prior to conception, psychopathology during pregnancy and early infants' development: A prospective-longitudinal study. Archives Women's Mental Health, 16, 549-560. http://dx.doi.org/10.1007/s00737-013-0376-5

Meijssen, D., Wolf, M. J., Koldewijn K., Van Baar, A. L., \& Kok, J. (2011). Maternal psychological distress in the first two years after very preterm birth and early interventions. Early Child Development Care, 181, 1-11.

Moraes, A. B, Beltrami, L., \& Souza, A. P. R. (2013). Ansiedade materna puerperal e risco para o desenvolvimento infantil. Distúrbios da Comunicação, 25(2), 229-239.

Nasreen, H. E., Kabir, Z. N., Forsell, Y., \& Edhborg, M. (2010). Low birth weight in offspring of women with depressive and anxiety symptoms during pregnancy: Results from a population based study in Bangladesh. BMC Public Health, 10 (515), 1-8. http://dx.doi.org/10.1186/1471-2458-10-515

Newham, J. J., Wittkowski, A., Hurley, J., Aplin, J. D., \& Westwood, M. (2014). Effects of antenatal yoga on maternal anxiety and depression: A randomized controlled trial. Depression and Anxiety, 31, 631-640. http://dx.doi.org/10.1002/da.22268

O'Connor, T. G., Monk, C., \& Fitelson, E. M. (2014). Practitioner review: Maternal mood in pregnancy and child development - Implications for child psychology and psychiatry. Journal of Child Psychology and Psychiatry, 55(2), 99-111. http://dx.doi.org/10.1111/jcpp.12153

Oliveira, E. A., Frizzo, G. B., \& Marin, A. H. (2000). Atitudes maternas diferenciais para com meninos e meninas de 4 e 5 anos. Psicologia: Reflexão e Crítica, 13(3),363-371

Padovani, F. H. P., Linhares, M. B. M., Carvalho, A. E. V., Duarte, G., \& Martinez, F. E. (2009). Avaliação de sintomas de ansiedade e depressão em mães de neonatos pré-termo durante e após hospitalização em UTI-Neonatal. Revista Brasileira de Psiquiatria, 26, 251-254.
Mariana Reichelt Chemello, Daniela Centenaro Levandowski e Tagma Marina Schneider Donelli

Padula, R. S., Pires, R. S., Alouche, R. S. Chiavegato, L. D., Lopes, A. D., \& Costa, L. O. P. (2012). Análise da apresentação textual de revisões sistemáticas em fisioterapia publicadas no idioma português. Revista Brasileira de Fisioterapia, 16(4), 281-288.

Paul, I. M., Downs, D. S., Schaefer, E. W., Beiler, J. S., \& Weisman, C. S. (2013). Postpartum anxiety and maternalinfant health outcomes. Pediatrics, 131(4), 1218-1224. http://dx.doi.org/10.1542/peds.2012-2147

Pérez, R. R. G., Bellót, Y. B., Pérez, Y. G., \& Herrera, B. B. (2013). Estrés y ansiedad maternos y su relación con el éxito de la lactancia materna. Revista Cubana de Pediatría, 86(2), 179-188.

Perosa, G. B., Ribeiro, D. G., \& Padovani, F. H. P. (2014). Mental health, mother-child interaction and development at the end of the first year of life. Paidéia, 24(59), 331-339. http://dx.doi.org/10.1590/1982-43272459201407

Perrone, R. A. P., \& Oliveira, V. B. (2011). Controle da ansiedade materna de bebê pré-termo via contato lúdicográfico. Estudos de Psicologia. (Campinas), 28(2), 269270.

Qiu, A., Rifkin-Graboi, A., Chen, H., Chong, Y-S, Kwek, K, Gluckman, P. D, Fortie M. V., \& Meaney, M. J. (2013). Maternal anxiety and infants' hippocampal development: timing matters. Translational Psychiatry, 3(1-7). http://dx.doi.org/10.1038/tp.2013.79

Rapoport, A., \& Piccinini, C. A (2011). Maternidade e situações estressantes no primeiro ano de vida do bebê. Psico-USF, 16(2), 215-225.

Rallis, R., Skouteris, H., McCabe, M., \& Milgrom, J. (2014). The transition to motherhood: Towards a broader understanding of perinatal distress. Women and Birth, 27(1), http://dx.doi.org/10.1016/j.wombi.2013.12.004

Reck, C., Noe, D., \& Gerstenlauer, J. (2012). Effects of postpartum anxiety disorders and depression on maternal self-confidence. Infant Behavior Development, 35(2), 264272. http://dx.doi.org/10.1016/j.infbeh.2011.12.005

Ribeiro, D. G., Perosa, G. B., \& Padovani, F. H. P. (2014). Fatores de risco para o desenvolvimento de crianças atendidas em Unidades de Saúde da Família, ao final do primeiro ano de vida: Aspectos sociodemográficos e de saúde mental materna. Ciência \& Saúde Coletiva, 19(1), 215-226.

Richter, N., \& Reck, C. (2013). Positive maternal interaction behavior moderates the relation between maternal anxiety and infant regulatory problems. Infant Behavior \& Development, 36(4), 498-506. http://dx.doi.org/10.1016/j.infbeh.2013.04.007

Rodrigues, C. L. (2011). Aspectos Neuropsicológicos dos Transtornos de Ansiedade na Infância e Adolescência: Um Estudo Comparativo entre as Fases de Pré e PósTratamento Medicamentoso. Dissertação de Mestrado, Universidade São Paulo, SP, Brasil. 


\section{- H* INTERACÃO EM L* PSICOLOGIA}

Romero-Gutiérrez, G., Rocha-Morales, D., \& Ruiz-Treviño, A. S. (2013). Resultados de la aplicación de la escala de Hamilton modificada en el diagnóstico de ansiedad materna durante el puerperio imediato. Ginecología y Obstetricia Mexico, 81, 180-185.

Sanchez, S. E., Puente, G. C., Atencio, G., Qiu, C., Yanez, D., Gelaye, B., \& Williams, M. A. (2013). Risk of spontaneous preterm birth in relation to maternal depressive, anxiety, and stress symptoms. Journal of Reproductive Medicine, 58, 25-33.

Schmidt, E. B., \& Argimon, I. I. L. (2009). Vinculação da gestante e apego materno fetal. Paidéia, 19(43), 211-220.

Silveira, F. C. P, Padovani, F. H. P., Perosa, G. B., Canavez, I. C., \& Peraçoli, J. C. (2009). Sintomas depressivos e ansiosos em mães de recém-nascidos com e sem malformações. Revista. Brasileira Ginecologia Obstetrícia, 31(9), 433-439.

Sherestha, S., Adachi, K., Petrini, M. A., \& Sherestha, S. (2014). Factors associated with post-natal anxiety among primiparous mothers in Nepal. International Nursing Review, 61, 427-434. http://dx.doi.org/10.1007/s00737013-0376-5
Mariana Reichelt Chemello, Daniela Centenaro Levandowski e Tagma Marina Schneider Donelli

Sockol, L. E., Epperson, C. N., \& Barber, J. P. (2014). The relationship between maternal attitudes and symptoms of depression and anxiety among pregnant and postpartum first-time mothers. Archives Women's Mental Health, 17(3), 199-212. http://dx.doi.org/10.1007/s00737-0140424-9.

Zelkowitz, P., Na, S., Wang, T., Bardin, C., \& Papageorgiou, A. (2011). Early maternal anxiety predicts cognitive and behavioural outcomes of VLBW children at 24 months corrected age. Acta Paediatrica, 100, 700-704. http://dx.doi.org/10.1111/j.1651-2227.2010.02128.

Recebido em: 01/04/2016 Primeira decisão editorial em: 28/07/2016 Aceito em: 10/10/2016 